\title{
Toward a rodent model of the Iowa gambling task
}

\author{
RUUD VAN DEN BOS, WILMA LASTHUIS, ESTHER DEN HEIJER, \\ JOHANNEKE VAN DER HARST, and BERRY SPRUIJT \\ Utrecht University, Utrecht, The Netherlands
}

\begin{abstract}
The Iowa gambling task in humans is, in principle, suited for the study of the long-term efficiency of behavior in a biologically relevant context. Key features of this task are uncertainty of outcomes and a conflict between the immediate and the long-term payoff options. Animal models allow us to study the underlying neurobiology of decision-making processes and the long-term efficiency of behavior in more detail and at a greater depth than is possible in humans. Therefore, we set out to develop a model of this task in rodents, using the task's key features. In this article, we describe the results of the first series of experiments with rats and mice. The data thus far suggest that mice and rats behave in a way similar to humans; that is, they tend to choose the option with the best long-term payoff more often as the test progresses.
\end{abstract}

So, there you are, dressed sharp as a knife, hungry, and ready to paint the town red. But at which restaurant will you satisfy your appetite today? At the little cozy cafe down at the harbor, where they serve normal-sized meals but ones that vary in quality; at the fancy restaurant on the other side of town, small meals but extremely tasty; or at the run-down joint a couple of blocks away, where you can get a large fatrich meal? But alas, the traffic is extremely heavy on this hot evening, and a long car drive does not really appeal to you. And, well, there was this nice waitress down at the harbor restaurant last week, and she said she might be coming this Saturday. Wow, and she was dressed to kill! And what about this new place that your friends told you about? This is the place to be; you have to check it out!

Everyone will recognize this kind of Saturday night deliberating, balancing costs and benefits between different options in a situation in which such costs and benefits vary. Our decisions do have long-term consequences even when we, for instance, simply satisfy our immediate needs day after day. Suppose that we did go more often to the restaurant down the block; we might become overweight over time. Thus, there is an inherent conflict between the immediate and the long-term payoffs of options. If we substituted a patch for restaurant or café, we would get essentially the same story for nonhuman animals - say, rats. Three stepping-stones have emerged over the last decade that allow us to study the long-term efficiency of the behavior of human and nonhuman animals in a coherent, biologically relevant framework, one of the key features of our research (Spruijt, van den Bos, \& Pijlman, 2001).

First, it has been shown in humans that decisions are not based on "cold," explicit reflections of all possible op-

We thank Nienke Valen for carrying out the experiments with male C57BL/6 mice. Correspondence concerning this article should be addressed to R. van den Bos, Faculty of Veterinary Medicine, Ethology and Welfare, Utrecht University, Yalelaan 2, NL-3584 CM, Utrecht, The Netherlands (e-mail: r.vandenbos@vet.uu.nl). tions. Rather, they are based on the concerted action of both emotional and cognitive processes, which are implicit, rather than explicit (Bechara, Damasio, Tranel, \& Damasio, 1997). These processes are related to structures common to human and nonhuman animals, such as the amygdala and the orbitofrontal cortex (Bechara, Damasio, Damasio, \& Anderson, 1994; Bechara, Damasio, Damasio, \& Lee, 1999; Bechara, Damasio, Tranel, \& Anderson, 1998; Bechara et al., 1997).

Second, a theoretical framework has been developed that seems to resolve the conflict between optimal and suboptimal foraging behavior in animals (Inglis, Langton, Forkman, \& Lazarus, 2001). It has been argued that animal behavior is driven by a conflict between keeping an eye on internal physiological factors, such as energy resources, and on external environmental factors, such as food sources in an environment that contains variations in, for example, resource quality. In the case of food, this leads to a tradeoff between moments of optimal foraging and suboptimal foraging. The latter means that the individual updates existing information or explores new areas in its surroundings, while giving less weight to how much food it will actually find. This tradeoff thus contains an element of self-control (cf. van den Bos, Houx, \& Spruijt, 2006).

Third, in humans, a test has been developed that captures some of the essential features of natural surroundings in which decision-making processes operate. In this test, the Iowa gambling task (IGT), subjects have to develop a longterm profitable monetary scenario in a situation of uncertainty concerning monetary outcomes and in the face of a conflict between the chance of encountering an immediate large reward in two long-term losing decks and the chance of encountering an immediate small reward in two longterm winning decks - that is, a conflict between immediate and long-term payoffs (Bechara et al., 1994). Figure 1 shows a typical result for this task (van den Bos, 2004).

Combined, these three stepping-stones allow us to understand the biological basis and context of long- 


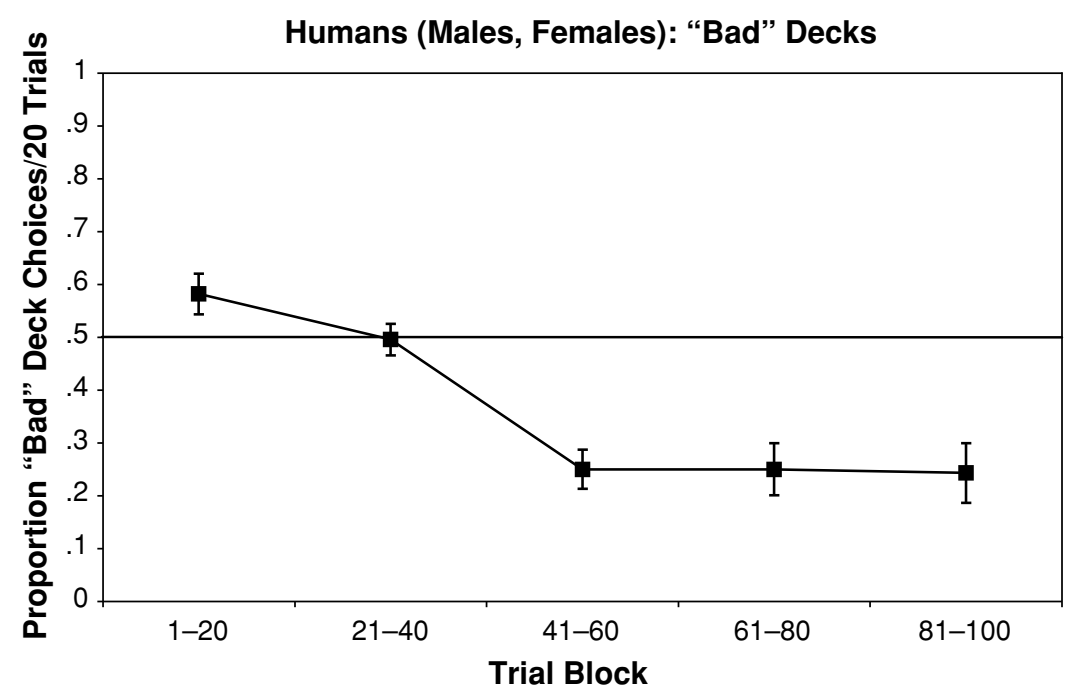

Figure 1. Example of the performance of the humans in the Iowa gambling task (van den Bos, 2004). Shown are the mean $( \pm S E M)$ proportions of choices for the "bad" decks $(A+B)$ per blocks of 20 choices for $n=22$ subjects $(n=16$ females and $n=6$ males; mean $[ \pm S E M]$ age, $36.3 \pm 2.4$ years).

term efficiency of behavior in both human and nonhuman animals, for which we have developed a working hypothesis in terms of two systems that regulate choices across time - that is, the (dopaminergic) reward system and the (serotonergic) cognitive control system (van den Bos, 2004; van den Bos et al., 2006). Thus far, no animal models exist of the IGT. Such models are crucial to understanding in more detail the underlying neurobiology of decision-making processes and how they might be regulated and/or disturbed by genetic, ontogenetic, and external factors. Therefore, we decided to develop a rodent (rat and mouse) model that captures the essence of the IGT in humans - that is, uncertainty of outcomes and a conflict between different options in terms of immediate and long-term payoffs. In this article, we report our results thus far, using rats and mice that were available in our laboratory at the time of testing. Thus, our goal is not to study, for instance, strain and gender differences in choice behavior in mice but, rather, to present the development of the model as such.

\section{METHOD}

\section{Subjects}

For the present experiments, both mice and rats were used. Male and female C57BL/6 and female DBA/2 were socially $(n=2-3)$ housed in Macrolon Type II cages with a shelter, paper shreds (Enviro Dry, Tecnilab, The Netherlands), and tissues as enrichments. They were either purchased from a commercial breeder (Harlan, Zeist, the Netherlands) or bred in our own laboratory. Male Wistar and Long-Evans rats were housed socially $(n=3)$ in Macrolon Type IV cages with a shelter and gnawing sticks (enriched housing) or with nothing (standard housing; see van der Harst, Baars, \& Spruijt, 2003, for a description). The rats were purchased from a commercial breeder (Harlan, Zeist, the Netherlands). All the rats were given tissues on a regular basis. Food was present ad lib, except for experimental periods. Water was freely available throughout. All the subjects were housed under a reversed 12:12-h day:night cycle (lights off at $0700 \mathrm{~h}$ ). Temperature was maintained at $21^{\circ}-23^{\circ} \mathrm{C}$. Humidity was $40 \%-50 \%$. A radio provided background noise throughout the day. All the experiments took place during the dark phase between 1000 and $1600 \mathrm{~h}$.

\section{Apparatus}

Mice. For the mice, an eight-arm radial maze was used (Figure 2A). The dimensions were as follows: diameter of the octagonal maze center, $22 \mathrm{~cm}$; wall height, $23.5 \mathrm{~cm}$; arm length, $22 \mathrm{~cm}$; arm width, $9.0 \mathrm{~cm}$; arm height, $5.0 \mathrm{~cm}$. Two arms were used as the start arm, and four as the goal arms (Figure 2A).

The mice were able to make a choice in the central octagonal area once they had left one of the start arms. We had noticed in pilot studies that mice were less accurate in their choices when the central area was used as start box, since they appeared to choose more often the arm where they happened to be at the very moment the octagonal slide door was lifted.

The walls of the central maze area were covered with white paper. This was done so that the mice would not be distracted and could not directly see the experimenter while he/she rebaited arms. We had noticed in pilot studies that without internal cues, mice could hardly differentiate goal arms, since no strong external cues were present to guide their choices. We therefore attached internal cues above the entrance of the arms, which helped the mice to differentiate the goal arms. These cues consisted of a cross or a circle $(5 \times 5 \mathrm{~cm})$, which was either black or white. Colors, rather than forms, were positioned adjacent to one another.

Rats. For the rats, a box in which we had carried out other gambling type experiments in the past was used (Figure 2B; van den Bos, Baars, Jonkman, \& Spruijt, 2003). It contained a start box, a choice area, and four "arms." The dimensions were as follows: start box, $60 \mathrm{~cm}$ long, $20 \mathrm{~cm}$ wide, and $59 \mathrm{~cm}$ high; choice area, $20.6 \mathrm{~cm}$ long, $61.9 \mathrm{~cm}$ wide, and $59 \mathrm{~cm}$ high; arm, $88.5 \mathrm{~cm}$ long, $15 \mathrm{~cm}$ wide, and $59 \mathrm{~cm}$ high.

On the basis of our experience with mice, we attached internal cues to the side of the walls of the arms, which helped the rats to differentiate the goal arms. These cues consisted of a cross or a circle $(10 \times 10 \mathrm{~cm})$, which was either black or white. Colors, rather than forms, were positioned adjacent to one another. 


\section{A}

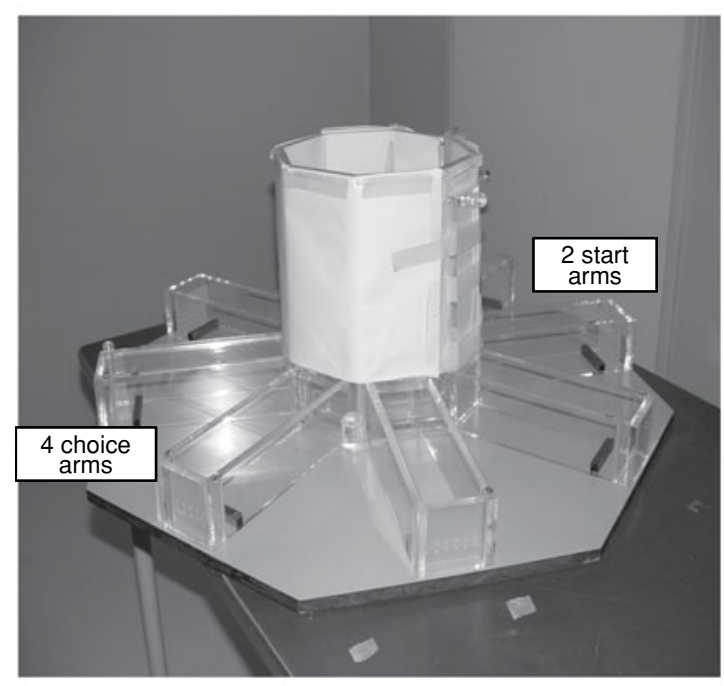

B

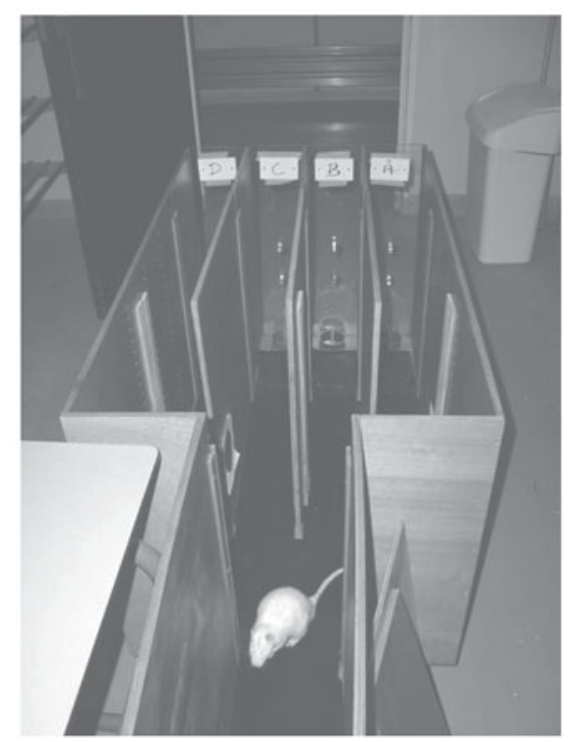

Figure 2. (A) Radial maze used for the experiments in mice. Two arms were used as the start arm (upper right corner; the box is positioned between the arms), and four were used as goal arms (lower left corner; the box is positioned between the arms). (B) Box used for the experiments in rats (see the text for details). Goal arms are labeled $\mathrm{A}-\mathrm{D}$; the start box is positioned at the lower end of the picture.

It should be noted that for the rats, an eight-arm radial maze (cf. the section on mice) would have been appropriate as well. As has been mentioned, the present box was used because we had used this box for other gambling type experiments (van den Bos et al., 2003). What is crucial for any apparatus is the fact that it should have a start box and four goal arms.

\section{Training Procedure}

For the mice and the rats, the training procedures were essentially the same. A trial was started by lifting the octagonal slide door of the center of the maze (mice) or the slide door of the goal box (rats).
Once the mice had hopped into one of the goal arms, the octagonal slide door was lowered, and the animals were allowed to sample the arm's contents. For the rats, a wooden panel was placed in front of the arm whenever they had made their choice, preventing them from leaving the arm.

To avoid the development of routines in mice, the order of start arms during sessions was randomized across trials. The goal arms differed in the extent to which they contained rewards (sugar pellets) and punishments (quinine-treated sugar pellets; see below for details).

Before the start of the first trial on each daily session, the mice and rats were confined for $30 \mathrm{sec}$ in one of the start arms (mice) or the start box (rats). The intertrial interval was $15 \mathrm{sec}$ thereafter. The animals were allowed to explore the arms and to eat the pellets for $2 \mathrm{~min}$ at maximum. Whenever the mice or rats did not leave the start arm or start box or did not make a choice within 2 min, the same trial was given again. Occasionally, the animals were gently pushed out of the start arm or start box. After each mouse, the floor of the maze was cleaned with $70 \%$ alcohol.

The mice or rats were given one habituation session (mice, $5 \mathrm{~min}$; rats, $10 \mathrm{~min}$ ) before the first experimental day. The mice were $6-8$ months old when tested; the rats were 4-6 months old.

Hungry male and female mice $(90 \% \pm 5 \%$ free-feeding weight) were given 15 trials a day over 9 days. Hungry male rats $(90 \% \pm 5 \%$ free-feeding weight) were given 10 trials a day for 12 days. The food regimen was such that the mice or rats received a specified amount of food between 1600 and $0700 \mathrm{~h}$ on the basis of free-feeding hourly consumption measures. During the weekend, they had ad lib access to food. Water was freely available throughout.

To avoid aggression between the mice, they were separated each day during testing and were released together in their home cage directly after their daily test session. For the rats, this was not necessary.

\section{"Gambling" in Mice and Rats}

Table 1 contains an overview of how the human IGT is translated into an animal model.

Money was represented by food. Monetary wins were represented by pieces of sweetened puffed wheat cereal (mice) or sugar pellets (rats), and losses were represented by unpalatable, but not uneatable, quinine-treated pieces of sweetened puffed wheat cereal (mice) or quinine-treated sugar pellets (rats).

The prearranged order of wins and losses per deck of cards was represented by a prearranged order of sugar and quinine pellets per

Table 1

Overview of the Relationship Between Human and Animal Gambling Tasks

\begin{tabular}{ll}
\hline Human Iowa Gambling Task & \multicolumn{1}{c}{ Animal Model (Rats/Mice) } \\
\hline Four decks of cards & four goal arms \\
Money & food \\
Monetary win & $\begin{array}{l}\text { sweetened puffed wheat cereal } \\
\text { pieces (mice)/sugar pellet (rats) }\end{array}$ \\
Monetary loss & $\begin{array}{l}\text { quinine-treated sweetened puffed } \\
\text { wheat cereal pieces (mice)/quinine } \\
\text { treated sugar pellet (rats) }\end{array}$
\end{tabular}

Position of wins/losses uncertain per 10 cards

position of sweetened cereal pieces/ quinine-treated sweetened cereal pieces, or sugar pellets/quininetreated sugar pellets, uncertain per prearranged 10 choices

Monetary conflict between immediate payoff (standard test, factor of 2)

Monetary conflict between long-term payoff per 10 cards ("good" and "bad"; standard test difference: $€ 500$ ) conflict between immediate big and small reward (mice, factor 3.3; rats, factor of 3)

conflict between amount of food per 10 choices due to quinine-treated rewards (difference: mice, $35 \mathrm{mg}$; rats, 5 pellets) 
arm. The uncertainty of the monetary wins and losses in the prearranged order of blocks of cards per deck (Bechara et al., 1994) was represented by a prearranged order of sweet and bitter rewards, in which the position of sweet or bitter rewards varied per block of 10 choices for each arm. Thus, there was no rule for the mice and rats to learn about when they would run into rewards or punishments.

"Bad" Decks A and B, which contain a chance of winning big monetary rewards ( $\$ 100$ or $€ 100$ ) but do have losses of $\$ 250$ or $€ 250$ per 10 cards (Bechara et al., 1994; van den Bos et al., 2006) were represented by arms in which, occasionally, a big reward (mice, 20-30 mg pellets; rats, three sugar pellets) was found among quinine-treated pellets (9/10 positions). "Good" Decks C and D, which contain a chance of obtaining small monetary rewards (50 US dollar/Euro) and do have overall wins of 250 US dollar/Euro per 10 cards (Bechara et al., 1994; van den Bos et al., 2006), were represented by arms in which, regularly, a small reward (mice, 5- to 10 -mg pellets; rats, one sugar pellet) was found among quininetreated pellets ( $2 / 10$ positions). The conflict between immediate (A and $\mathrm{B}$ ) and long-term (C and D) payoffs is modeled by differences in the amount of food obtained per arm; for the mice, for the immediate payoff in $\mathrm{A}$ and $\mathrm{B}$ versus $\mathrm{C}$ and $\mathrm{D}$, there was a difference by a factor of 3.3, and for the long-term payoff in $\mathrm{C}$ and $\mathrm{D}$ versus $\mathrm{A}$ and $\mathrm{B}$, there was a difference by a factor of 2.4 per 10 positions $(35-\mathrm{mg}$ difference between "good" and "bad" arms); for the rats, for the immediate payoff in $\mathrm{A}$ and $\mathrm{B}$ versus $\mathrm{C}$ and $\mathrm{D}$, there was a difference by a factor of 3, and for the long-term payoff in $\mathrm{C}$ and $\mathrm{D}$ versus $\mathrm{A}$ and $\mathrm{B}$, there was a difference by a factor of 2.7 per 10 positions (five-pellet difference between "good" and "bad" arms).

To check for aspecific effects, we made a variant with two empty arms and two rewarded (one "good"/one "bad") arms.

Quinine-treated pieces of sweetened puffed wheat cereal or sugar pellets were made by mixing pieces of sweetened puffed wheat cereal (Honey Smacks, Kelloggs) or sugar pellets (BioServ Inc., Frenchtown, NJ) with a $180-\mathrm{mM}$ quinine (Sigma-Aldrich, Schnelldorf, Germany) solution for a few minutes. They were subsequently dried in the air on paper towels. This procedure led to small and hard brownish pieces for the mice and small quinine/sugar pellets for the rats. Hereafter, they were stored in small vessels and shielded from light until use. These pellets were unpalatable but not uneatable, as judged by the behavior of the mice and rats. It should be noted that they occasionally showed the quinine-specific responses, such as drooling, chin rubbing, and so forth (Berridge, 1996).

Before the experiments started, the mice and rats were accustomed to the pieces of sweetened cereal (mice) or sugar pellets (rats). This occurred by introducing the rewards in their home cages, 1 week prior to experimentation. Subsequently, it was checked whether the mice or rats ate the rewards by assessing their consumption in a separate cage (mice, Macrolon Type II; rats, Macrolon Type IV).

Sugar/sweetened cereal and quinine-treated sugar pellets/pieces of sweetened cereal were placed at the end of the arm behind a small black barrier in the case of the mice and in a small cup (diameter, $6 \mathrm{~cm}$; height, $1 \mathrm{~cm}$ ) in the case of the rats.

Rewarded and empty arms, as well as "good" and "bad" arms, were counterbalanced across subjects to avoid bias in the experimental design.

\section{Analysis and Dependent Variables}

Exclusion criteria, for the mice and rats alike, were (1) subjects that did not reliably eat the rewards, (2) subjects that did not leave or hardly left the start box or did not make a choice while in the choice area, and (3) subjects that ate quinine pellets (see the Results section).

It was noted down which arm the subject entered and whether it ate the sweet or bitter rewards or not. When only "good" and "bad" arms were present, only the proportion of visits to the "bad" arms per daily number of trials (mice, 15 trials; rats, 10 trials) was calculated. When empty $(n=2)$, and "good" $(n=1)$ and "bad" $(n=1)$, arms were used, two proportions were calculated: the proportion of visits to the empty arms per daily number of trials (mice, 15 trials; rats, 10 trials) and the proportion of visits to the "bad" arms per number of visits to rewarded arms.

Statistical analysis was done with a two- or three-way ANOVA with one factor as the repeated measure (session; see the Results section for the factors). Post hoc testing was done as appropriate. All the analyses were run in SPSS Versions 9.0 and 10.0 for PC. Differences were considered to be significant when $p \leq .05$. There was considered to be a trend when $.05<p \leq .10$. Results were considered not significant when $p>.10$ (n.s.). All statistics are two-tailed unless otherwise indicated.

\section{RESULTS}

\section{Mice: Gender Differences}

Figure $3 \mathrm{~A}$ shows the performance of male and female mice in the variant with two "good" and two "bad" arms. Male mice tended to choose "good" arms more often than did females across days [two-way ANOVA, gender $\times$ session (repeated measure): session, $F(8,64)=3.357, p \leq .003$; gender $\times$ session, $F(8,64)=1.841, p \leq .086]$.

On the basis of these results, we decided to test whether female mice would be more apt to discriminate the rewarded arms of the maze when two arms were left empty, while one arm was used as "good" arm and one as "bad" arm. The remainder of the protocol was left the same. Three male mice were discarded, since they did not eat the rewards regularly. Figure $3 \mathrm{~B}$ and $3 \mathrm{C}$ show the results. Independently of gender, mice more often chose the rewarded arms across sessions [two-way ANOVA, gender $\times$ session (repeated measure): session, $F(8,80)=9.438$, $p \leq .001$; session $\times$ gender, $F(8,80)=0.372$, n.s.; gender, $F(1,10)=0.138$, n.s.]. However, a close inspection of Figure 3C shows that toward the end of the test, the males chose the "good" arms overall more often than did the females, which was confirmed by statistical analysis [Sessions 5-9: $F(1,10)=15.602, p \leq .003$ ].

\section{Mice: Strain Differences}

Figures $4 \mathrm{~A}$ and $4 \mathrm{~B}$ show the results of female DBA/2 and $\mathrm{CB} 57 \mathrm{BL} / 6$ mice. Both DBA/2 mice and C57BL/6 mice gradually chose the rewarded arms more often across sessions [two-way ANOVA, strain $\times$ session (repeated measure): session, $F(8,136)=9.682, p \leq .001$; strain $\times$ session, $F(8,136)=0.391$, n.s.]. However, DBA/2 mice tended to choose the "good" arms less often than did the C57BL $/ 6$ mice as the test progressed [session, $F(8,136)=$ $2.310, p \leq .024$; session $\times$ strain, $F(8,136)=1.877, p \leq$ $.068]$.

\section{Rats: Strain and Housing Conditions}

A number of rats were discarded from the analysis. First, 6 rats were discarded because they did not leave the start box and/or did not eat the sugar pellets. It should be noted that this concerned only Long-Evans rats, especially those from the enriched condition (5/6). Second, 6 rats were discarded from the analysis because they consumed the quinine pellets. Especially Wistar rats $(n=5)$ did so. No bias with respect to housing conditions existed.

Figures $5 \mathrm{~A}$ and $5 \mathrm{~B}$ show the results of the rat experiments. With respect to the empty arms, the rats appeared 


\section{A Male-Female C57BL/6 Mice: "Bad" Arms}

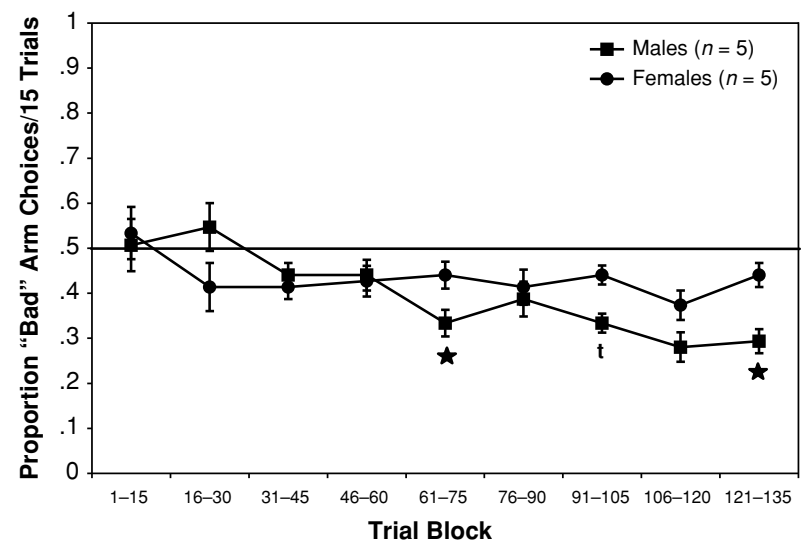

B Male-Female C57BL/6 Mice: Empty Arms

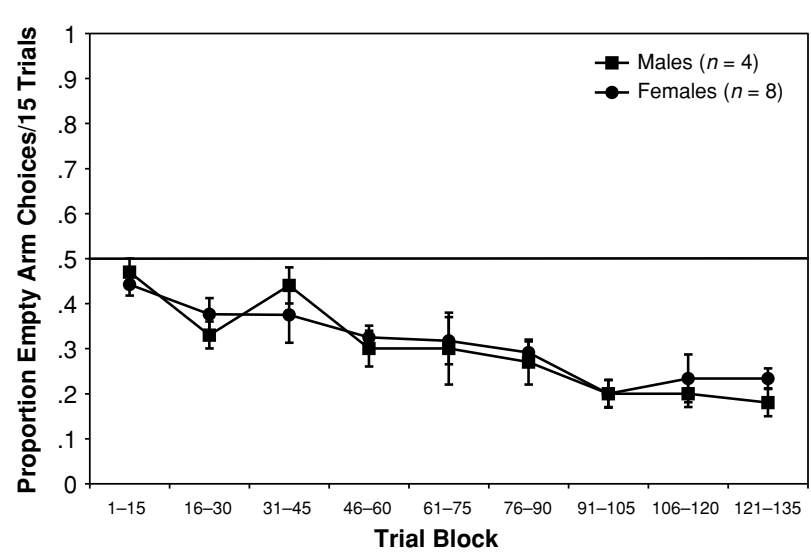

C Male-Female C57BL/6 Mice: "Bad” Arms

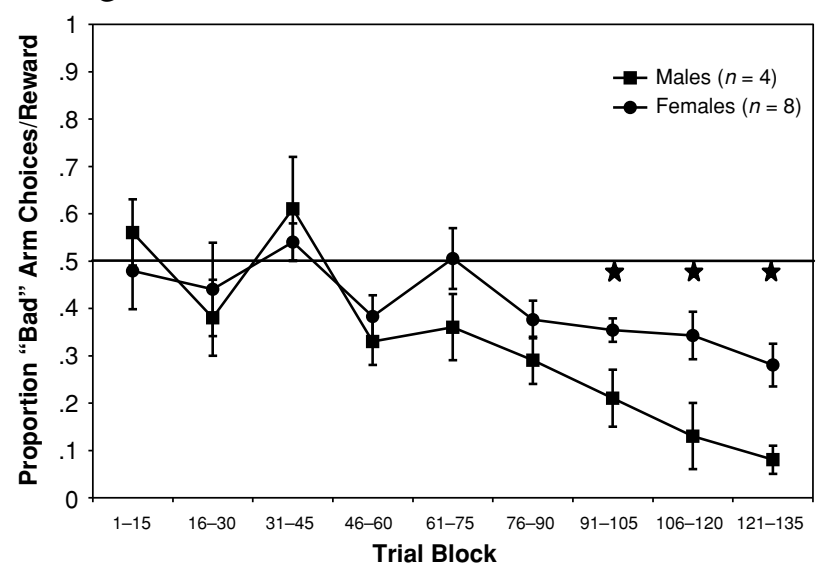

Figure 3. (A) Performance of male and female mice in the mouse Iowa gambling task (IGT) model. Shown are the mean ( $\pm S E M$ ) proportions of choices for the "bad" arms per block of 15 choices of $n=5$ females and $n=5$ males. $t: p \leq .10$. " $p \leq .05$ ( $t$ test for independent samples). (B) Performance of male and female mice in the mouse IGT model. Shown are the mean ( $\pm S E M)$ proportions of choices for the empty arms per block of 15 choices of $n=8$ females and $n=4$ males. (C) Performance of male and female mice in the mouse IGT model. Shown are the mean $( \pm S E M)$ proportions of choices for the "bad" arms per block of 15 choices of $n=8$ females and $n=4$ males. $\quad * p \leq .05$ ( $t$ test for independent samples).

to choose more often the rewarded arms across sessions [three-way ANOVA, strain $\times$ housing $\times$ sessions (repeated measure): session, $F(11,220)=12.074, p \leq .001]$ regardless of strain [strain $\times$ session, $F(11,220)=1.460$, n.s.], housing condition [housing $\times$ session, $F(11,220)=$ 0.821 , n.s.], or their combination [session $\times$ housing $\times$ strain, $F(11,220)=1.036$, n.s.]. On the whole, however, Wistar rats chose rewarded arms more often than did LongEvans rats [strain, $F(1,20)=5.760, p \leq .026$ ], regardless of housing conditions [strain $\times$ housing condition, $F(1,20)=0.559$, n.s.].

With respect to the rewarded arms, the rats appeared to choose "good" arms more often across sessions [threeway ANOVA, strain $\times$ housing $\times$ sessions (repeated measure): session, $F(11,220)=7.599, p \leq .001]$, regardless of strain [strain $\times$ session, $F(11,220)=1.195$, n.s.], housing condition [housing $\times$ session, $F(11,220)=$ 0.575 , n.s.], or their combination [session $\times$ housing $\times$ strain, $F(11,220)=0.357$, n.s.]. On the whole, however,
Wistar rats chose "good" arms more often than did LongEvans rats [strain, $F(1,20)=11.904, p \leq .003$ ], regardless of housing conditions [strain $\times$ housing condition, $F(1,20)=0.003$, n.s.].

\section{DISCUSSION}

The present study was a first attempt to develop a rodent model for the IGT in humans. The data thus far indicate that mice and rats, like humans, do seem to choose the best long-term option more often as the test progresses although differences do exist between gender and strains.

At first sight, the learning curves for humans (Figure 1), mice (Figures 3 and 4), and rats (Figure 5) with respect to choosing the option with the best long-term payoff are quite similar. Initially, all individuals do sample the different options in order to choose more often the "good" arms in the long run. It is clear, however, that more experiments are needed to determine whether this similarity in choice 
A Female C57BL/6-DBA Mice: Empty Arms

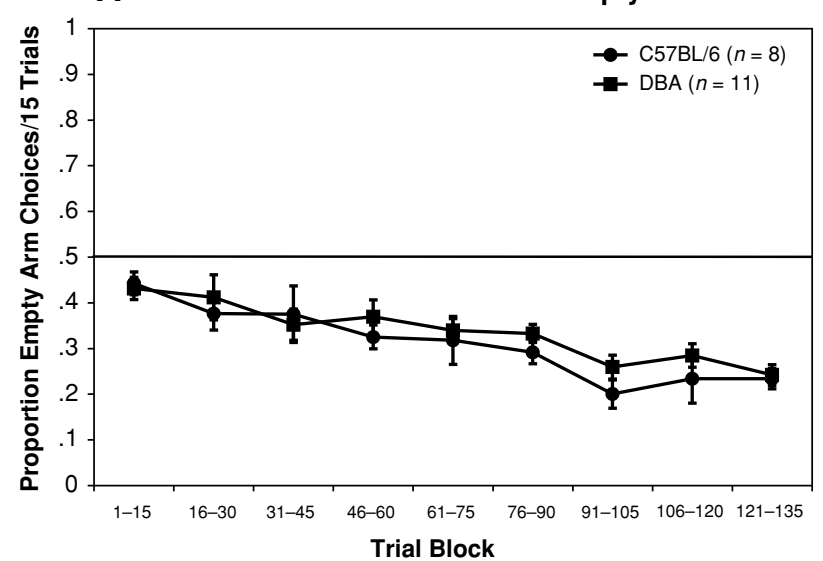

B Female C57BL/6-DBA Mice: "Bad” Arm

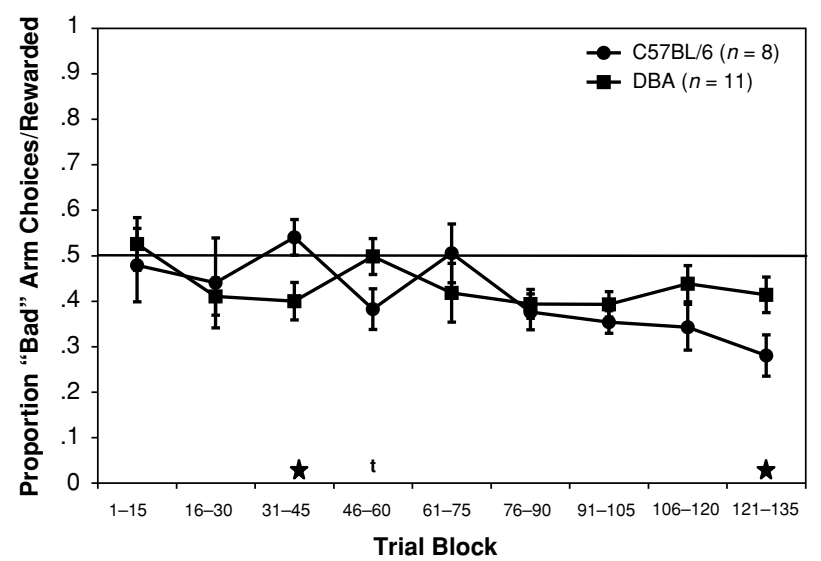

Figure 4. (A) Performance of female mice of the C57BL/6 and DBA/2 strain in the mouse Iowa gambling task (IGT) model. Shown are the mean $( \pm S E M)$ proportions of choices for the empty arms per block of 15 choices of $n=8$ female C57BL/6 mice and $n=11$ female DBA/2 mice. (B) Performance of male and female mice in the mouse IGT model. Shown are the mean $( \pm S E M)$ proportions of choices for the "bad" arms per block of 15 choices of $n=8$ female C57BL/6 mice and $n=11$ female DBA/2 mice. $t: p \leq .10$. " $p \leq$ .05 ( $t$ test for independent samples).

behavior of humans, on the one hand, and rodents, on the other, is more than just superficial. Accordingly, the next series of experiments will be directed at studying the underlying neurobiology in rats and mice in more detail, to reveal whether they are equally sensitive to lesions in key structures, such as the amygdala and orbitofrontal cortex (Bechara et al., 1994; Bechara et al., 1999; Bechara et al., 1998; Bechara et al.,1997) and whether they are equally sensitive to changes in the experimental paradigm (van den Bos et al., 2006).

A few observations on the present data set deserve further discussion.

First, the female mice tended to choose the "good" arms less often than did the male mice, which was not due to a deficit in spatial learning and/or cue discrimination per se, as shown by the fact that the females and the males were equally likely to choose the empty arms less often as the test progressed. When we plotted the individual learning curves of the males and females, we noticed that the individual learning curves of the females showed far more day-to-day variation than did the learning curves of the males; that is, whereas the female mice did repeatedly choose the "good" arm during the one day, they did so less often during the next (data not shown). One explanation for this difference in performance between the female and the male mice might be sought in the effect of the estrus cycle on reward sensitivity and in the neurotransmitter systems involved in this test. It has been shown, at least in humans, that the rewarding value of sweet items fluctuates across the menstrual cycle (Cabanac, 1979). Furthermore, it has been shown and argued that the dopaminergic and serotonergic systems are crucial for performing the test (Bechara, Damasio, \& Damasio, 2001; van den Bos et al., 2006), both of which show strong fluctuations across the estrus cycle in humans and rodents (Becker, 1999; Dluzen \& Ramirez, 1985; Fernández-Ruiz, Hernandez, Demiquel,
\& Ramos, 1991; Ho, Olsson, Westberg, Melke, \& Eriksson, 2001; Justice \& de Wit, 1999; Nördstrom, Olsson, \& Halldin, 1998; Rubinow, Schmidt, \& Roca, 1998; Shimizu \& Bray, 1993; Thompson \& Moss, 1997). It should be noted that in humans, it has been shown that males and females differ in their performance on the IGT: Males tend to choose on the basis of long-term outcome, whereas females tend to choose on the basis of both immediate wins and long-term outcomes (Overman et al., 2004; Reavis \& Overman, 2001). These data seem to be in line with the present data. However, thus far, menstrual cycle effects have not unequivocally demonstrated in humans (Reavis \& Overman, 2001; van den Bos, den Heijer, Houx, \& Spruijt, 2006). Future experiments will be directed at studying these gender and menstrual/estrous cycle differences in more detail in humans and rodents.

Second, the mice of the DBA/2 strain appeared to choose the "good" arms less often than did the mice of the $\mathrm{C} 57 \mathrm{BL} / 6$ strain. Although it has been reported that $\mathrm{DBA} / 2$ mice are poorer spatial learners than $\mathrm{C} 57 \mathrm{BL} / 6$ mice (Rossi-Arnaud, Faglioli, \& Ammassari-Teule, 1991), this does not seem to account for the present data, since we did not observe a difference with respect to the empty arms. In fact, given that the arms are cued, DBA/2 should actually perform better than C57BL/6 mice, since they are reported to be better cue learners (AmmassariTeule, Passino, Restivo, \& de Marsanich, 2000). Thus, it may be hypothesized that the difference is due to a difference in sensitivity to rewards. Indeed, DBA/ 2 mice are, in general, less sensitive to rewards than are C57BL/6 mice (Lush, 1988; Misra \& Pandey, 2003). Furthermore, it has been shown that DBA/2 mice are more sensitive than C57BL/6 mice to food-restriction-induced sensitization of the meso-accumbens dopamine system (Cabib \& Bonaventura, 1997), which leads to their tolerating costs for a longer period of time (Salamone \& Correa, 2002; Spruijt 

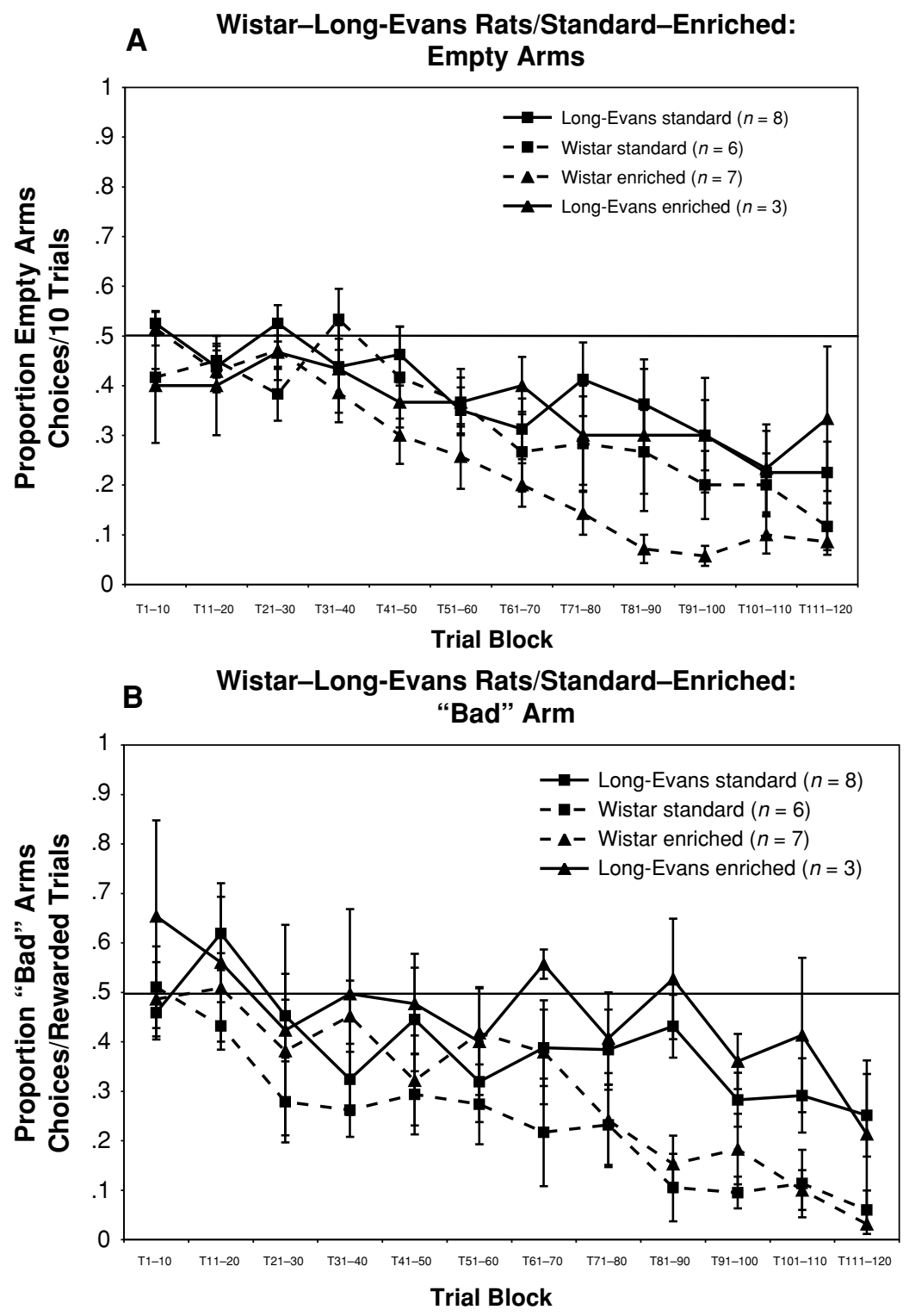

Figure 5. (A) Performance of male rats of the Wistar and Long-Evans strains housed under either standard or enriched conditions in the rat Iowa gambling task (IGT) model. Shown are the mean $( \pm S E M$ proportions of choices for the empty arms per block of 10 choices of $n=8$ standard and $n=3$ enriched housed Long-Evans rats and of $n=6$ standard and $n=7$ enriched housed Wistar rats. (B) Performance of male rats of the Wistar and Long-Evans strains housed under either standard or enriched conditions in the rat IGT model. Shown are the mean $( \pm S E M)$ proportions of choices for the "bad" arms per block of 10 choices of $n=8$ standard and $n=3$ enriched housed Long-Evans rats and of $n=6$ standard and $n=7$ enriched housed Wistar rats.

et al., 2001), expressed in this task as an increased number of visits to the "bad" arms (van den Bos et al., 2006). Alternatively, one may hypothesize that DBA/2 mice explore the options more thoroughly than do C57BL/6 mice, which, in turn, decide on the basis of less information and may, therefore, be labeled as impulsive in this sense (see Cabib, Puglisi-Allegra, \& Ventura, 2002; van den Bos, 2004; van den Bos et al., 2006). It is clear that this needs further study (see also below).
Third, in contrast to what we observed in mice, we observed differences between rat strains - that is, Wistar and Long-Evans rats - that were not due specifically to differences in reward sensitivity but to more general differences in exploratory strategies. Along with the results of the mice experiments, this shows the added value of using empty arms, since they allow us to differentiate specific reward-related effects from more general effects. The question now arises whether Long-Evans rats 
are poorer learners than Wistar rats. It has been shown in the Morris water maze that Long-Evans rats show a better performance than do Wistar rats and behave much like wild rats (Harker \& Whishaw, 2002). Furthermore, they have been suggested to pay more attention, and more thoroughly so, to their environment than do Wistar rats (Ambrogi Lorenzini, Bucherelli, Giachetti, \& Tassoni, 1987; Tonkiss, Shultz, \& Galler, 1992). We observed in the present experiments that Wistar rats often ran at high speed from the start to the goal box, whereas Long-Evans rats moved more slowly and explored their environment while on their way (data not shown). Thus, given these data, one may argue that Wistar rats are more impulsive or more reward prone than are Long-Evans rats. Alternatively, Long-Evans rats have been suggested to be less sensitive to rewards, such as ethanol (Gauvin, Moore, \& Holloway, 1993). Thus, they may pay less attention to the reward arms in general, and to the "good" arm in particular. Future experiments will be directed at analyzing these differences in more detail.

Fourth, a number of Wistar rats ate quinine-treated pellets throughout the experiment. We tested the rats in four batches, and especially the animals in the last batch behaved in this way. Prior to the last batch, we prepared new quinine/sugar pellets and noticed that they appeared to be slightly less bitter than the previous ones. Accordingly, the balance between sweet and bitter is crucial to whether rats, especially Wistar rats, will consume the pellets or not.

Fifth, in previous experiments, it has been shown that standard housed Wistar rats are more willing to exert effort for rewards than are enriched housed Wistar rats (van der Harst et al., 2003). In the present experiment, we did not observe such a strong housing effect, except that enriched housed Long-Evans rats were not willing to perform in the experiment.

In conclusion, the present study is a first step toward developing an animal analogue of the IGT. The setup we have chosen seems promising, since we observe a profile in choice behavior that resembles that in humans.

\section{REFERENCES}

Ambrogi Lorenzini, C., Bucherelli, C., Giachetti, A., \& Tassoni, G. (1987). Spontaneous and conditioned behavior of Wistar and Long Evans rats. Archives Italiennes de Biologie, 125, 155-170.

Ammassari-Teule, M., Passino, E., Restivo, L., \& de Marsanich, B (2000). Fear conditioning in C57/BL/6 and DBA/2 mice: Variability in nucleus accumbens function according to the strain predisposition to show contextual- or cue-based responding. European Journal of Neuroscience, 12, 4467-4474.

Bechara, A., Damasio, A. R., Damasio, H., \& Anderson, S. W. (1994). Insensitivity to future consequences following damage to human prefrontal cortex. Cognition, 50, 7-15.

Bechara, A., Damasio, H., \& Damasio, A. R. (2001). Manipulation of dopamine and serotonin causes different effects on covert and overt decision-making. Society of Neuroscience Abstracts, 27, 465.5.

Bechara, A., Damasio, H., Damasio, A. R., \& Lee, G. P. (1999). Different contributions of the human amygdala and ventromedial prefrontal cortex to decision-making. Journal of Neuroscience, 19, 5473-5481.

Bechara, A., Damasio, H., Tranel, D., \& Anderson, S. W. (1998). Dissociation of working memory from decision making within the human prefrontal cortex. Journal of Neuroscience, 18, 428-437.

Bechara, A., Damasio, H., Tranel, D., \& Damasio, A. R. (1997).
Deciding advantageously before knowing the advantageous strategy. Science, 275, 1293-1295.

BeCKer, J. B. (1999). Gender differences in dopaminergic function in striatum and nucleus accumbens. Pharmacology Biochemistry \& Behavior, 64, 803-812.

BERRIDGE, K. C. (1996). Food reward: Brain substrates of wanting and liking. Neuroscience \& Biobehavioral Reviews, 20, 1-25.

Cabanac, M. (1979). Sensory pleasure. Quarterly Review of Biology, 54, 1-29.

Cabib, S., \& Bonaventura, N. (1997). Parallel strain-dependent susceptibility to environmentally-induced stereotypies and stressinduced behavioral sensitization in mice. Physiology \& Behavior, 61, 499-506.

Cabib, S., Puglisi-Allegra, S., \& Ventura, R. (2002). The contribution of comparative studies in inbred strains of mice to the understanding of the hyperactive phenotype. Behavioural Brain Research, 130, 103-109.

Dluzen, D. E., \& Ramirez, V. D. (1985). In vitro dopamine release from the rat striatum: Diurnal rhythm and its modification by the estrous cycle. Neuroendocrinology, 41, 97-100.

Fernández-Ruiz, J. J., Hernandez, M. L., Demiquel, R., \& Ramos, J. A. (1991). Nigrostriatal and mesolimbic dopaminergic activities were modified throughout the ovarian cycle of female rats. Journal of Neural Transmission, 85, 223-229.

Gauvin, D. V., Moore, K. R., \& Holloway, F. A. (1993). Do rat strain differences in ethanol consumption reflect differences in ethanol sensitivity or the preparedness to learn? Alcohol, 10, 37-43.

HaRKer, K. T., \& WhishaW, I. Q. (2002). Place and matching-to-place spatial learning affected by rat inbreeding (Dark-Agouti, Fischer 344) and albinism (Wistar, Sprague-Dawley) but not domestication (wild rat vs. Long-Evans, Fischer-Norway). Behavioural Brain Research, 134, 467-477.

Ho, H.-P., Olsson, M., Westberg, L., Melke, J., \& Eriksson, E. (2001). The serotonin reuptake inhibitor fluoxetine reduces sex steroid-related aggression in female rats: An animal model of premenstrual irritability? Neuropsychopharmacology, 24, 502-510.

Inglis, I. R., Langton, S., Forkman, B., \& Lazarus, J. (2001). An information primacy model of exploratory and foraging behavior. Animal Behaviour, 62, 543-557.

Justice, A. J. H., \& DE Wit, H. (1999). Acute effects of $d$-amphetamine during the follicular and luteal phases of the menstrual cycle in women. Psychopharmacology, 145, 67-75.

Lush, I. M. (1988). The genetics of tasting in mice: VI. Saccharin, acesulfame, dulcin and sucrose. Genetics Research, 53, 95-99.

Misra, K., \& PANDEy, S. C. (2003). Differences in basal levels of CREB and NPY in nucleus accumbens regions between C57BL/ 6 and DBA/2 mice differing in inborn alcohol drinking behavior. Journal of Neuroscience Research, 74, 967-975.

Nördstrom, A., Olsson, H., \& Halldin, C. (1998). A pet study of $\mathrm{D}_{2}$ dopamine receptor density at different phases of the menstrual cycle. Psychiatry Research, 83, 1-6.

Overman, W. H., Frassrand, K., Ansel, S., Trawalter, S., Bies, B., \& Redmond, A. (2004). Performance on the IOWA card task by adolescents and adults. Neuropsychologia, 42, 1838-1851.

Reavis, R., \& Overman, W. H. (2001). Adult sex differences on a decision-making task previously shown to depend on the orbital prefrontal cortex. Behavioral Neuroscience, 115, 196-206.

Rossi-Arnaud, C., Faglioli, S., \& Ammassari-Teule, M. (1991). Spatial learning in two inbred strains of mice: Genotype-dependent effect of amygdaloid and hippocampal lesions. Behavioural Brain Research, 45, 9-16.

Rubinow, D. R., Schmidt, P. J., \& Roca, C. A. (1998). Estrogenserotonin interactions: Implications for affective regulation. Biological Psychiatry, 44, 839-850.

Salamone, J. D., \& Correa, M. (2002). Motivational views of reinforcement: Implications for understanding the behavioral functions of nucleus accumbens dopamine. Behavioural Brain Research, 137, 3-25.

Shimizu, H., \& Bray, G. A. (1993). Effects of castration, estrogen replacement and estrus cycle on monoamine metabolism in the nucleus accumbens, measured by microdialysis. Brain Research, 621, 200-206.

Spruijt, B. M., van den Bos, R., \& PiJlman, F. T. A. (2001). A concept of welfare based on reward evaluating mechanisms in the brain: 
Anticipatory behavior as an indicator for the state of reward systems. Applied Animal Behaviour Science, 72, 145-171.

Thompson, T. L., \& Moss, R. L. (1997). Modulation of mesolimbic dopaminergic activity over the rat estrous cycle. Neuroscience Letters, 229, 145-148.

Tonkiss, J., Shultz, P., \& Galler, J. R. (1992). Long-Evans and Sprague-Dawley rats differ in their spatial navigation performance during ontogeny and at maturity. Developmental Psychobiology, 25 567-579.

VAN DEN Bos, R. (2004). Emotion and cognition. In M. Bekoff (Ed.), The handbook of animal behavior (pp. 554-557). Westport, CT: Greenwood.

van den Bos, R., BaArs, A., Jonkman, S., \& SpruiJt, B. (2003). Costbenefit assessment in rats. Acta Neurobiologiae Experimentalis, 63, S49.
VAn den Bos, R., Den Heijer, E., Houx, B., \& Spruijt, B. (2006, July). The Iowa Gambling Task and the menstrual cycle: Type of reward effects? [Abstract]. Paper presented at the FENS Forum, Vienna.

VAN Den Bos, R., Houx, B. B., \& Spruit, B. M. (2006). The effect of reward magnitude differences on choosing disadvantageous decks in the Iowa gambling task. Biological Psychology, 71, 155-161.

VAN DER Harst, J. E., BAARs, A. M., \& SpruiJT, B. M. (2003). Standard housed rats are more sensitive to rewards than enriched housed rats as reflected by their anticipatory behavior. Behavioural Brain Research, 142, 151-156.

(Manuscript received October 5, 2005; revision accepted for publication January 14, 2006.) 